\title{
Web Based System For ChoOSE OPTIMAL Micro Modules TeChNOLOGical VARIANT
}

\author{
YORDANOV, R. \& TODOROV, R.
}

Abstract: In this paper we are going to present a design and implementation of web based system used to aid engineers for choose optimal technological variant, during design of hybrid micro modules. We rely on well recognized open source platform for development of enterprise solutions, such as Java EE. The selected architecture is plug-in orientated and based on one of the popular standard for modular software OSGI. As base we are using the prouven mathematical algorythms for calculatin film resistors, capacitors and cols combined with multivariable optimization process, in respect of both thick and thin layered production technologies.

Key words: MCM, multivariable optimization
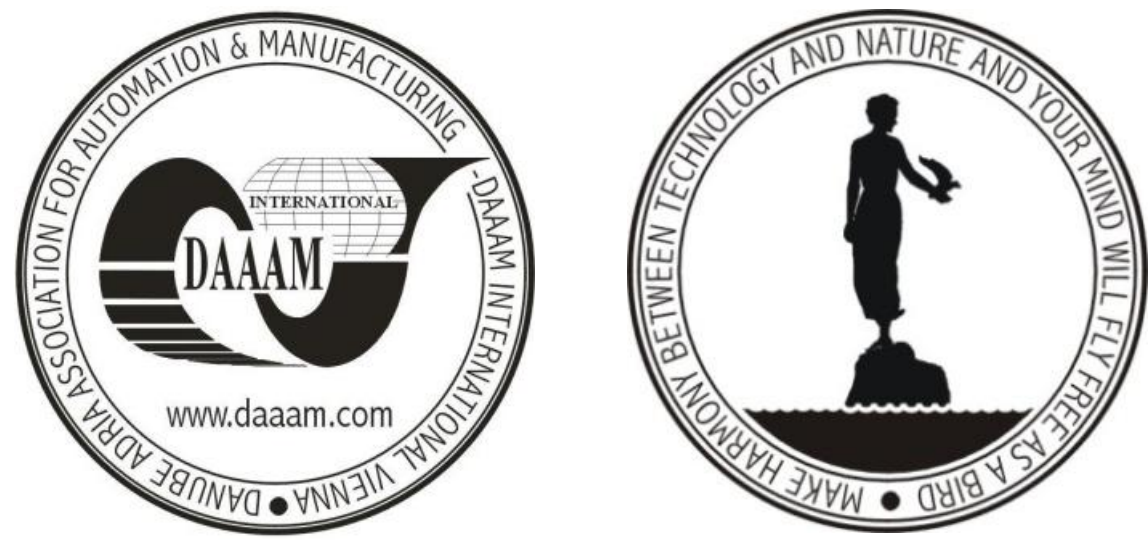

Authors' data: Ass.Prof. Yordanov, R[umen]; Todorov, R[umen], Technical University of Sofia, 8, Kliment Ohridski St. Sofia-1000, Bulgaria, yordanov@tusofia.bg, rtodorov@csc.com

This Publication has to be referred as: Yordanov, R[umen] \& Todorov, R[umen] (2010). Web Based System for Choose Optimal Micro Modules Technological Variant, Chapter 65 in DAAAM International Scientific Book 2010, pp. 753-766, B. Katalinic (Ed.), Published by DAAAM International, ISBN 978-3-901509-74-2, ISSN 1726-9687, Vienna, Austria

DOI: $10.2507 /$ daaam.scibook.2010.65 


\section{Introduction}

Technology's thin film resistors, resistors, capacitors and coils are formed by lithographically patterning on the surface of a wide variety of substrate materials. The process of selecting the best combination of these materials during design of hybrid micro circuits is valuable engendering works which requires big amount of engineering skills, experience and effort. For doing this job the engineering stuff needs to have appropriate tolls which can aid this process. The scientific base for doing this job is well known and it consist combination of theoretic engineering methodologies with experimental proven notations and also depends on the designers experience and the statistical data they have on their previous efforts. On other hand Internet has proven as outstanding environment for cooperation, and also as environment which highly increase the accessibility. The available software solutions solving engineering task are mainly desktop based systems, which must be installed on a specific computer with specific operation system. There are some successful implementations of functionality common to desktop applications presented in web. Some companies have achieved significant results like Goole and their web provided service - Google Docs. This inspired us to try to create an engineering solution following their philosophy to present everything in web without any requirements for the user to install software in its local machine. The main problem we are trying to solve is to create a web based system which can aid engineers and students in the process of selection best combination of materials during a hybrid micro-module design. We have decided to use the web based approach because we are trying to improve the accessibility to the system within a university or a company private network. After is installed on a central server, the system can be access only via web browser and no software need to be installed. As a part of our research, in this paper, we are aiming to share our experience in developing such system, in our university, which can assist engineers and students during their everyday work. For developing this university project we rely on well known open source solutions. The leading platform we use is Java EE 1.5 in collaboration with some other open source solutions. Some of the system modules rely on algorithms which can be adjusted or improved and we have decided to use plug-in orientated approach for these modules. As foundation we rely on the popular OSGI platform, which can provide sophisticated plug-in management support. To achieve the required dynamic behaviour by the user interfaces we use combination of Java Server Faces and Ajax technologies to keep the behaviour as close as possible to a desktop system. The system performs calculation of film resistors, capacitors and coils using well known and proven design algorithms and methodologies. Also it aids the engineer to perform multi parametric optimization process for selecting best combination of conductive, nonconductive materials in combination with substrates for selecting best combination needed for creating well suited hybrid micro module. All results are presented in a table view and the process of ordering is made according set input variables which weight is adjustable. As a result we are going to share the initial impression about how the students as users accept such no traditional approach. 


\section{Theoretical Basis}

This is a short theoretical review in some of the most important concepts relying in the process of hybrid micro-modules design and the multivariable optimization process. In the next several pages we are going to do a short review to the most commonly used technologies for hybrid micro-module design. After that we are going to introduce in short the most common way for designing film resistors, capacitors and inductors which is one of the tasks our application is performing. The theoretical overview will end with some basic aspects of the multivariable optimization process, used for selecting the best combination of technological variants.

\subsection{Thick-film technology}

The thick-film technology consists an additive process that utilizes screen printing methods to apply conductive, resistive, and insulating films, initially in the form of a viscous paste, onto a ceramic substrate in the desired pattern. The films are subsequently dried and fired at an elevated temperature to activate the adhesion mechanism to the substrate.

\subsection{Thin-Film Materials}

Thin-film technology, in contrast to thick-film technology, is a subtractive technology in that the entire substrate is coated with several layers of material and the unwanted material is etched away in a succession of photoetching processes. The use of photolithographic processes to form the patterns enables much narrower and more well-defined lines than can be formed by the thick-film process. This feature promotesthe use the thin-film technology for high-density and high-frequency applications.

\subsection{Thin-Film versus Thick-Film}

Reviewing the differences in general we can say that thin-films and thick films do not complete witch each other. In many areas these are very complementary and sometimes displaying different advantages in different applications. Many applications require the combination of bath technologies to achieve proper circuit performance.

Thin-films have certain properties and characteristics:

- Thin-film resistors have superior temperature coefficient and are more stable and have a lower noise

- Thin-films resistors and circuits can be made smaller because of the photolithographic processing

- They have well defined lines with smooth ages, low surface roughness, and dance pure material compositions

- Thin-films wire bonds more easily and with greater consistence

Thick-films provide properties and advantages: 
- Thick films are typically patterned by screen printing, although etched thick films are used for precision line creation

- Thick film links can also be dispensed through a moving capillary to direct write a pattern

- Thick films form an excellent multilevel circuits and crossovers because of the thickness and quality of their fired dielectric layers

- Resistors are available in a wider range of values

- Thick-film conductor materials have higher resistivity, but the inherently greater volume of the thick film conductor leads to low-resistance conductors.

- Simple thick-film circuits are lower in cost than thin films.

The catalogues provided by the material supplier provide good set of information about material characteristics. There are also many comprehensive guides about film materials which can be used as reference. For example a good start could be Ohring, 2002.

\subsection{Film Resistors Design}

One of the important features of the application is the possibility do the initial calculations which are needed for a film resistor design. In general, there are four parameters of concern to the engineer when designing a resistor; the resistor value, its change in value over time, its change in value with temperature, and its power handling capability. As with any type of resistor, total resistance is determined by the equation:

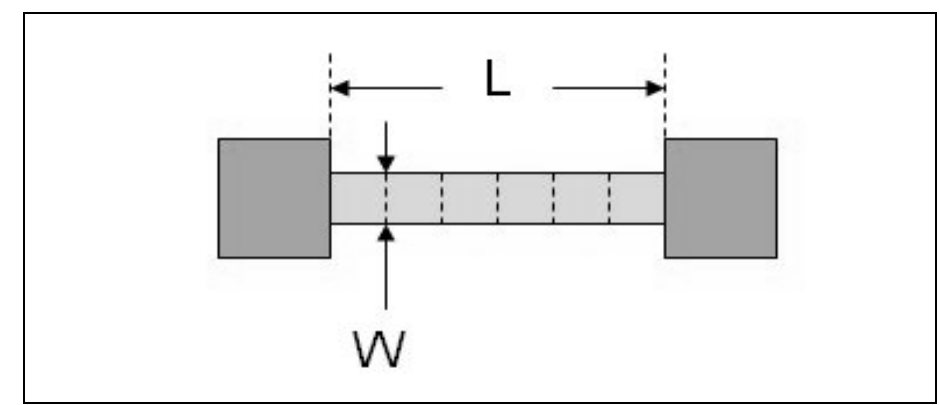

Fig. 1. Rectangular film resistor

$$
R=\frac{\rho_{B} L}{W T}
$$

where

$\mathrm{R}=$ electrical resistance,

$\rho_{B}=$ bulk resistivity of the material, ohms-length

$\mathrm{L}=$ length of the sample in the appropriate units

$\mathrm{W}=$ width of the sample in the appropriate units

$\mathrm{T}=$ thickness of the sample in the appropriate units

With thin film resistors, sheet resistance in units of ohms per square $(\Omega / \square)$ is often used to specify a film and is calculated as: 


$$
R_{\text {sheet }}=\frac{\rho}{T}
$$

where: $\square=$ Unit Surface Area of Equal Length and Width

This approach permits easy calculation of thin film resistor values as follows:

$$
R_{\text {total }}=R_{\text {sheet }} \frac{L}{W}
$$

It is important to identify the quantity:

$$
\frac{L}{W}
$$

It defines the number of "squares" that a resistor has, and total resistance is proportional to number of squares. Note that squares have no units.

When:

$$
\frac{L}{W}>1
$$

The leading is $\mathrm{L}$ and the shape simmilar to the one shown in figure 1 .

Sometimes this equation has diferent scale:

$$
\frac{L}{W}<1
$$

In this case the leading dimension is $\mathrm{W}$ and in this case this quiatity is transformed to:

$$
\frac{W}{L}
$$

Fig. 2. Rectangular film resistor shape with W/L lesser than 1

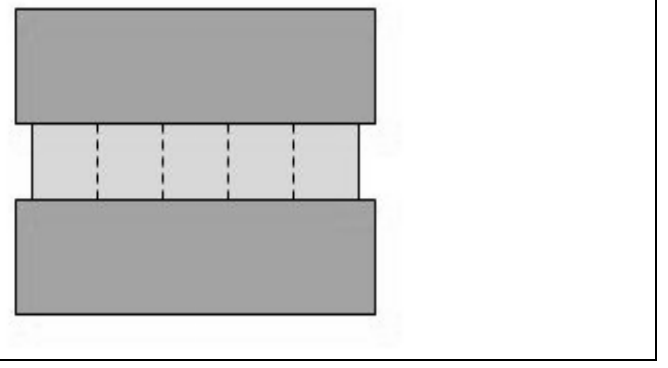


In case when:

$$
\frac{L}{W}>10
$$

This is a coomon case when more complex shapes are introduced:

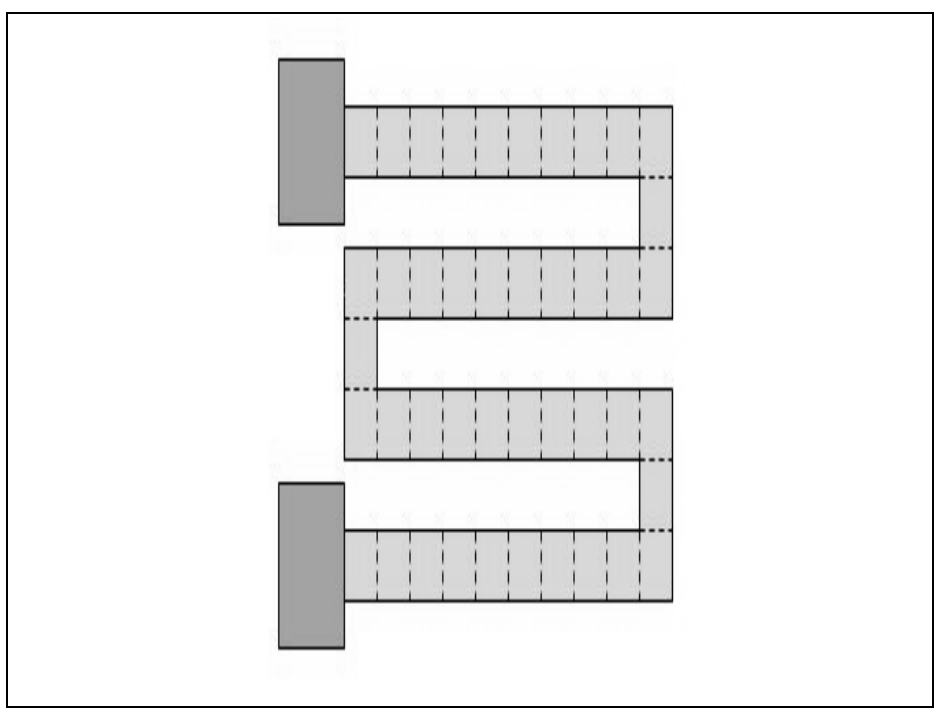

Fig. 3. Meander resistor shape

Basically, a meander resistor can be considered as a bar resistor with the exception of the corner squares (right-angle bends). Due to the change in current density at right-angle path, the effective number of squares is 0.559 , which is commonly used in resistor design. Because of limmitation of the technology available, such complex shapes are rearly aplicable for thick film resistors.

\subsection{Film Capacitors Design}

Thick film capacitors have usually three layer parallel plate structure. This is shown in a figure 4.

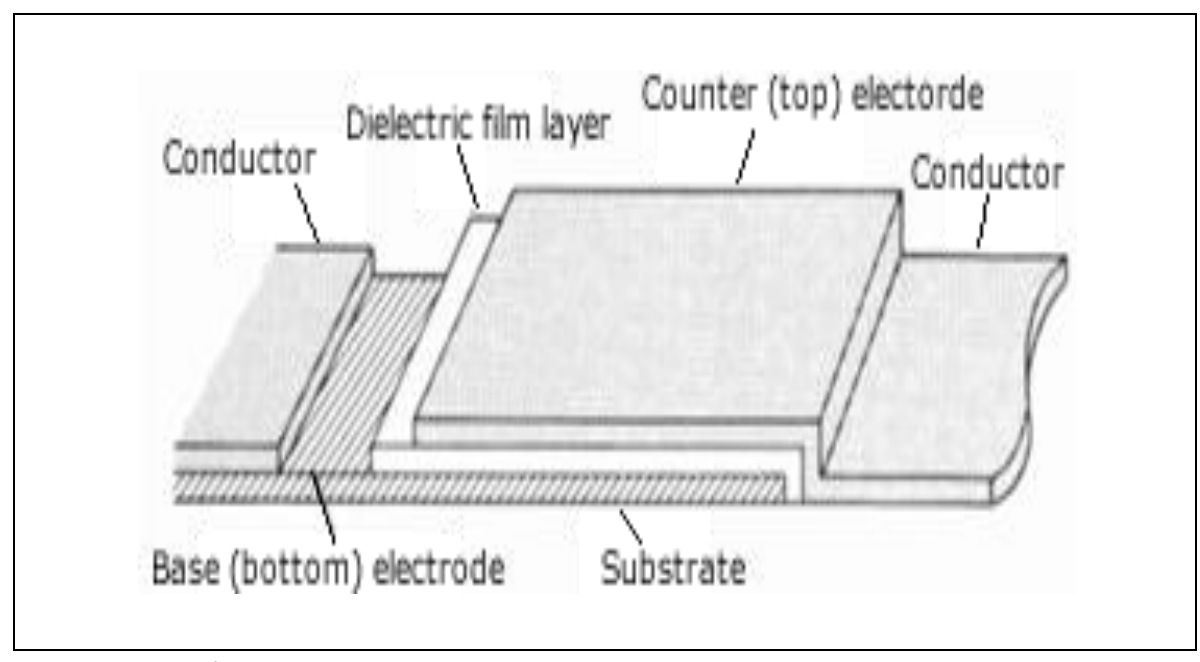

Fig. 4. Substrate capacitor 
The effective capacitor area $\mathrm{A}$ is determined by the electrode overlap. The lowfrequency capacitance is given by:

$$
C=\frac{\varepsilon_{o} \varepsilon_{r} A}{d}
$$

where :

$\varepsilon_{o}$ - permittivity of free space

$\varepsilon_{r}$ - relative dielectric constant of the given material

$d$ - dielectric film thickness.

One of the common design parameters for the dielectrics is the breakdown voltage, $V_{B}$ which is related to the dielectric material breakdown strength, $E_{B}$ for the bulk.

$$
V_{B}=\frac{E_{B}}{d}
$$

However for thin films, $E_{B}$ tends to be less than the breakdown strength of bulk material. Thus the dielectric breakdown strength for a film $E_{B F}$, is always less than the breakdown strength $E_{B}$ of the bulk. Hence, $d$ for a given $V_{B}$ of the film must be greater than would normally be calculated for the bulk. Because the complexity and variety of mechanism for dielectric breakdown, especially in films, the working voltage $V_{R}$ and the associated field strength, $E_{R}$ for thin-film capacitors are some fraction of the bulk breakdown values, that is:

$$
V_{R}=K V_{B}=K E_{B} d
$$

Here $\mathrm{K}$ is constant in value between 0 and 1.The coefficient $\mathrm{K}$ is chosen for reliable cooperation.

Thin capacitors are not ideal charge storage elements, because of losses of the dielectric, electrodes and the leads. Their shape also parallel plated capacitors cannot be trimmed.

To reduce the lead resistance in capacitance structure without suffering the value of $\mathrm{C}$, a new type film capacitor is introduced - interdigital capacitor. It consists of two metal strips with interleaved metal fingers on a common substrate above a full coverage ground. There is capacitance between each finger and the ground plane, as well as mutual capacitance and inductance between the fingers.

In a low frequency mode the, capacitance is approximately given by the formula:

$$
C=\frac{\left(\varepsilon_{r}+1\right) \varepsilon_{o} L}{W}\left[(n-3) K_{1}+K_{2}\right]
$$


where $n$ is the number of fingers of length $L, W$ is the width of the pattern, $K_{1}$ is the contribution of the interior fingers and $K_{2}$ is the contribution of the exterior finger.

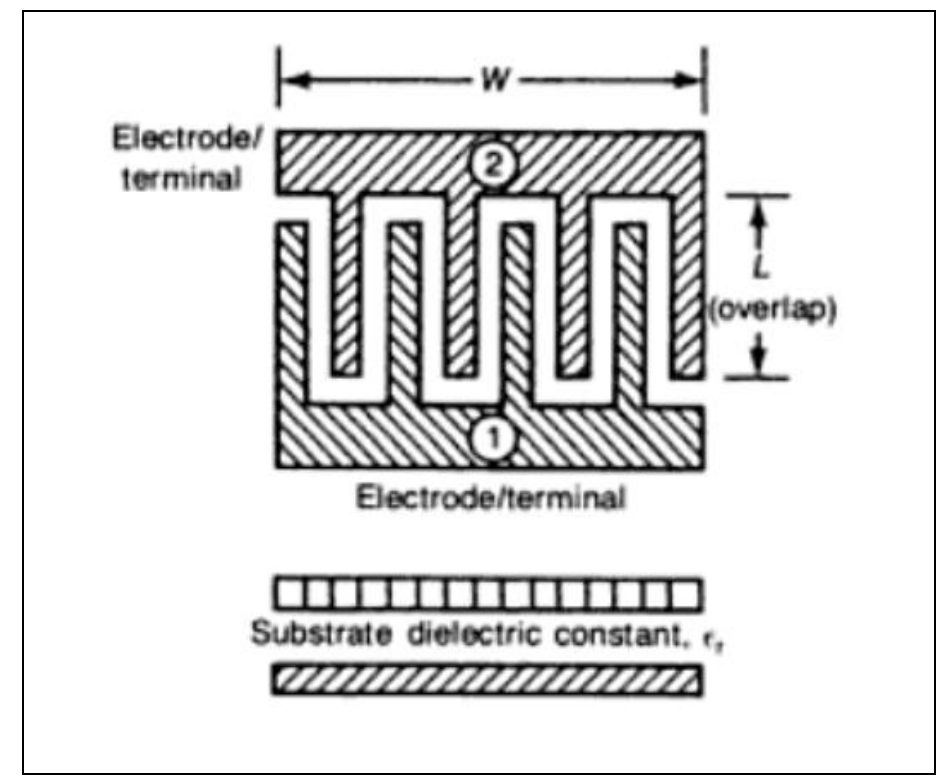

Fig. 5. Thin film interdigital capacitor

\subsection{Film Inductors Design}

Thin film inductors are usually made by strip of conductors.

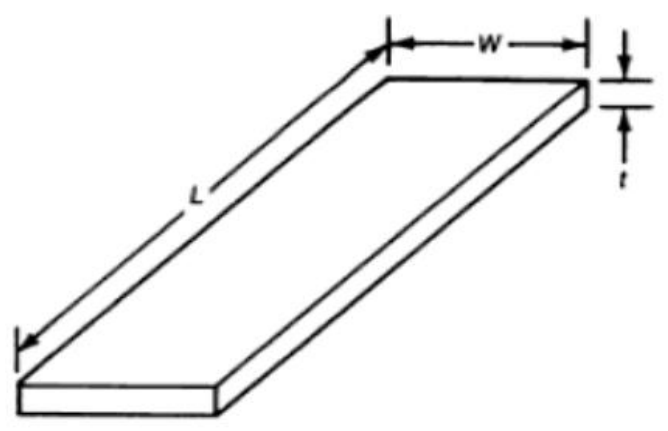

Fig. 6. Thin film strip inductor

The inductance per unit length of a plane single strip inductor is approximately as:

$$
L=0.005\left[\ln \left(\frac{l}{W+t}\right)+1.19+0.22 W+\frac{t}{l}\right]
$$

Where $W$ is the ribbon width, $l$ is the length of the unit, and $t$ is the film thickness. The resistance of the inductor is:

$$
R=\frac{\rho}{2(W+t) \delta}
$$


Where $\rho$ is the resistivity and $\delta$ is the frequency dependent skin depths. Thus:

$$
Q=\frac{2 \pi f}{k} \frac{1}{R}
$$

Where $\mathrm{k}$ is a function $\mathrm{W} / \mathrm{t}$ and ranges $1<\mathrm{k}<2$. If more inductance is needed a spiral inductor can be formed. Thin film inductors are usually made by strip of conductors.

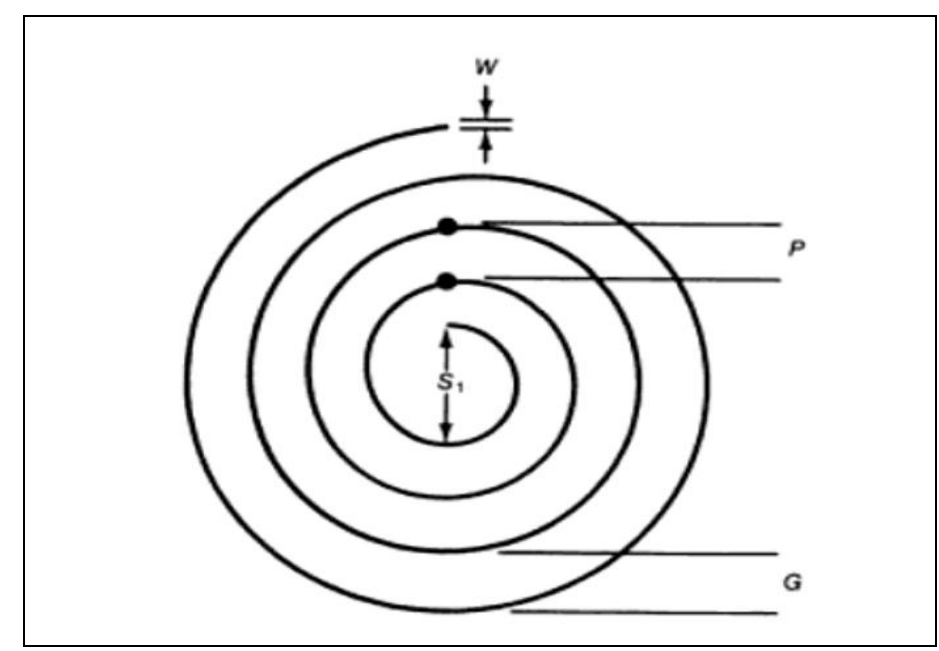

Fig. 7. Thin film strip inductor

A comprehensive information about the design of film discrete elements can be found in (Licari \& Haber, 1999) and (Whitaker, 2005).

\subsection{Multivariable Optimization Process}

The need to find minima / maxima of multivariable functions arises surprisingly often in practical engineering applications. The whole topic of system identification hinges around finding the best mathematical function representation of a system that is consistent with a series of experimental measurements of a number of outputs of the system. For more detailed explanation of the concepts of multivariable optimization see (Cooke et al., 1991).

\section{Detailed System Description}

\subsection{Requirements Overview}

The process of design of hybrid micro modules is an engineering effort which involves the engineer to choose between huge amounts of specialized materials and utilities and techniques. The process also enforces to make compromises in order to choose the best suitable solution. The right selection of materials is the most significant aspect in this process. In this case the researcher has to choose depending on a list of available parameters and the weight of these parameters depends on the current task. As we have mentioned in chapter 1, the process of designing film resistors, capacitors and coils within the micro module requires producing an image 
with specific shape over a previously prepared substrate. The combination of similar materials suitable for producing film resistors, combined with a set of materials suitable for capacitors and coils in a group is called technological variant. Every technological variant could be used in certain circumstances to be a source of material combination which the engineer can use. The source for the micro module is an electrical circuit which defines the types of discrete elements which should be constructed using film technology. Our aim was to create a suitable software solution for managing the technological variants and select the most suitable solution for a circuit used as input, according to set of variable requirements, which weight is adjustable depending on the needs of the chip designer. The traditional way of making such system is to create a desktop based application for certain operation system. We have chosen to use more different approach and made this system a web application. There is a trend for creating more and more complex web solutions in now days. The gap in functionality between desktop and web system was huge a decade ago. But in the last five years this gaps was made smaller thanks to a new introduction of technologies, especially AJAX (Asynchronous JavaScript and XML). Also as base we have decided to create this system truly open sources and to use only open source software technologies as building blocks for our system.

\subsection{Similar systems available}

There are huge software and enterprise solutions for aiding electronics engineers. There are systems which have become an industrial standard for the chip designers. There are multiple deep and significant researches in this field. There are huge corporations providing solutions in this field like Synopsys and Cadence. The resources involved in all this truly exceed our amount of resources and we do not try to compete. The significant point in our research is not to create revolutionary solution. Our aim was to create a solution base on a well known and standard scientific basis but using an innovative approach in result presentation. In our basic forecast we didn't find many similar systems which are web base system and solve such engineering problems.

\subsection{Provided functionality}

We have created a web based application which can aid a chip designer to do the prior selection of technological variant for an electrical circuit used as input. The application stores a set of predefined technological variants. The user has the possibility to manage these technological variants. As we have said before a technological variant contains three groups of film materials. The first group contains only film materials for resistors. These materials have similar characteristics but each of this material is suitable for producing resistors with different range of resistance. The same can be said for the groups of capacitors and coils. Sometimes the technological variant can be materials which are grouped by the material manufacturer and specified as a group of materials suitable to be used in combination. The functionality of managing technological variants involves the operations of adding, deleting and modifying materials within the variant. Also it provides the functionality of adding new technological variant or modifying the 
common data for existing variants. The user has the possibility to add schematic description. A series of discrete elements description can be added. These elements are used when it is not possible to construct some of the elements of the circuit with the film materials described in each group. This corresponds to the engineering approach to use external elements inside the chip when it is not possible to achieve good parameters with used combination of material. The next most important functionality that the system provides is the ability to design film resistors, capacitors and coils. This is done using previously described theoretical base. The produced resistors capacitors and coils are drawn in a same surface in favor with the calculated dimensions and the proportions of the elements. This is done for all every combination of suitable materials within a technological variant. The design result contains the combination of materials ordered according a set of coefficients which define the weight of each parameter. The weight of each weight coefficient is defined by the user as input. Also there is some additional typical to the web systems functionality like user management and e-mail notifications.

\subsection{Implementation and used technologies}

The system is built on the top the Java EE platform, which is a leading open source platform for developing enterprise web application. The system architecture is a standard three tier architecture provided on the base of the components provided by the platform. The data is stored in a relational database which is common for a web application. The database we use is Postgres SQL. This is one of the most sophisticated open source databases available which makes it ideal for our solution. For separation from the database we use the JPA (Java Persistence API) which creates a layer between the application and the database. The technology is common object relation mapping and gives us the possibility to manage the technological variants, electrical circuits, weight coefficients and all other stuff which needed to be stored as object called entities. Useful information about JPA can be found in (Keith $\&$ Schincariol, 2006). These objects are persisted in the database when needed. This technology is well known in Java community and it is started to be widely used. The business layer is created by Enterprise Java Beans 3.0. This is the last stable revision of this technology which is supported by the newsiest versions of most widely used java application servers. It provides us with server side components which encapsulate a common part of logic. Our components for storing and retrieving data are EJB 3.0 stateless session beans. One of the important features of this technology is the declarative transaction mechanism. The transactions are managed by Java 1.5 annotations added to the methods of the bean which should be executed within transaction. This helps us to manage out transaction more easily. For the process of designing film elements we use a separate module. This module is EJB 3.0 message driven bean (MDB). A good starting reading about EJB 3.0 is (Brooke \& MonsonHaefel, 2006) The main reason for using such kind of element is the need to have a non blocking user interface during the design process, which may require a big amount of time in some specific cases. The communication between the client and the message driven bean is done by configurable queues. Three queues are used by the system. The first one is the incoming queue which is used to send messages from 
the client to the MDB. When the data is processed by the bean, it stores it inside an outgoing queue. Because of the need of more sophisticated message processing by the user interfaces, we have changed the standard JMS Resource Adapter provided by the server with Apache Active MQ. The project Apache Active MQ is open source project provided by Apache Software Foundation to provide additional functionality to the Java Message Service (JMS) technology, part of which are the MDB. For receiving messages from the outgoing queue we are using Message Servlet. It is provided by Active MQ and allows for web clients to be notified, with a JavaScript callback, when a message is send to the queue. The calculation results are sent to the client when the calculation is done and immediately displayed. This is done with the Ajax API provided by Apache Active MQ. Because of the fact that the calculation algorithms can be changed and modified in certain amount of time we have decided to use a plug-in management for the calculation algorithms. The plug-in infrastructure is based on the OSGI standard. We are using the Equinox OSGI management system on which is based the popular Java development platform eclipse. Empowered with Equinox we are able easily to install and uninstall plug-ins. The OSGI plug-in system relies on bundles, which are typical .jar files with extended manifest, providing the possibility to contain child .jar files. Every bundle is loaded in separate class loaded so the jars inside it could not interfere with the jars in other bundles. For using Equinox in web application there are two approaches - embed Equinox in web container or embed an application inside Equinox web container. For our application we use the first approach. For achieving this goal we use the OSGI bridge project. It is add on to Equinox OSGI platform and provides forwarding an external HTTP request to internal base OSGI Http Service. The user interface we use to manage plug-ins is another open source project called Apache Felix Web Console. Apache Felix is an OSGI management system similar to Equinox, but we do not use it. We have retrieved the console made for Felix and with a little configuration; we made it available for Equinox inside a OSGI bridge. All algorithms are realized as OSGI bundles and installed inside Equinox with the help of Apache Fleix Web Console. This console also provides a web interface which allows dynamically installing or uninstalling plug-ins during runtime. The user interface is created by using the Java Server Faces (JSF) technology. It provides an infrastructure for creating dynamic web pages with a set of server side controls like menus, buttons, text fields, trees and others. We have chosen this technology because it can dramatically increase the development time of user interface. Because JSF is a standard and the reference implementation is very poor of components we have switched to the Apache Software Foundation implementation of this technology, a project called MyFaces. It provides a rich component set and it is one of the oldest and most stable implementations available. The set of components within MyFaces we are using is called Tomahawk. It has good amount of components and it is also one of the oldest and stable component sets. Because not all of the components were available we have created some custom ones, like the color picker component, we have created because it was not provided by Tomahawk. Because of the need to have reusable web page parts like common header and footer, distribution of one menu within multiple pages, we have decided to use a template system called Facelets. This 
is an extension to Java Server Faces technology provided by Java.net open source community. Using it we save a lot of effort because most of the common pages fragments were reusable. For adding more rich behavior we are using the JQuery JavaScript library. It provides a very nice set of objects for JavaScript DOM manipulation, events, effects and other. We also have used it to create progress bars and other time dependent components. The combination of these technologies helped us to create a rich user interface without too much effort. Of course there were several pitfalls coming from configuration problems and integration between the different technologies.

\section{Conclusion}

We have discovered huge amount of available open source technologies and solutions and we have used them to build a full scale system. In present we have all the technologies necessary for building sophisticated user interface similar to desktop systems. There are good Ajax base components sets and Ajax libraries for fast user interface development. There are some drawbacks like compatibility with old browsers so user which do no use Internet Explorer 5.0+ or Mozilla Firefox 2.x+ but this is no a problem concerning our research, this is a fundamental problem which is available for all systems. We have partially solved this by doing browser detection during web site request. In the case the user is not bale to display the components which use the newest technologies we redirect the user to an version of the system supporting old browser but with less dynamical user interface. Some performance issues appeared but mostly the execution time depends on the server configuration and also on the server hardware.

As a result of our research we have integrated this system in our university and we are going to observe the reaction of the students, which are going to be future engineers, to this different approach. As a matter of the current status of this research we can share our experience that student don't find any difficulties to use web system instead of desktop. The feedback is somehow positive. Sometimes there are people who don't like the fact that some of the tasks are executed slower that they expect. Some people have previous experience with desktop systems and expect to have everything at the moment. But this reaction is not always an issue and certain optimizations can be done and, as we said before, with a better hardware and bandwidth this won't be a problem. With further measurements and improvements all this drawbacks can be removed and as a result we are able to produce results almost similar to desktop system.

One possible farther development is to make the whole system plug-in orientated not just the math algorithms and technology selection module as it is done at the moment. In this case a plug-in can contain a user interface fragment. In this case installing specific plug-in will change the controls available to the user and is going to make the system more flexible. Of course this will introduce some complications about how the JSF pages will be compiled as parts of OSGI bundles. One of the reasons we don not introduced this in the first version is that the available tools and technologies for developing web application within OSGI are relatively 
new and not so stable and there is some lack of required functionality. Fortunately the OSGI Alliance and multiple independent vendors make a lot of efforts to improve this so in next bunch of years all the necessary functionality will be provided by the OSGI based platforms. Also the system can be a base to integrate more sophisticated decision algorithms.

Another UI representation can be developed using, for example, a desktop client to the system backend. Also the Java Web Start technology can be applied here. This will help to provide downloadable web system with desktop capabilities. Such approach is used in some systems and especially for software which data should be updated often. One of the drawbacks of this approach is that the user should have JRE installed on its system. In some institutions and universities the users do not have the rights to do often installations of new software and this can cause a problem. This problem could be partially solved by using Adobe Flex for creating the system user interface. The benefits here come by the fact that almost every modern browser comes with direct support of Flash player and flash technology. According this overview we can say that there is a huge basis for farther researches in this field and also multiple possibilities for improvements and future developments.

\section{References}

Brooke, B. \& Monson-Haefel, R. (2006). Enterprise JavaBeans 3.0, O'Reilly, 1005 Gravenstein Highway North Sebastopol CA 95472

Cooke N.;Adams H.;Dell P. \& Moore T. (1991). Basic Mathematics for Electronics, McGraw-Hill, 0028008537, 11 New Fetter Lane London EC4P 4EE

Geary, D. \& Horstmann, C. (2007). Core Java Server Faces - 2nd edition, Prentice Hall, 4150 Network Circle, Santa Clara, California 95054 U.S.A.

Keith M. \& Schincariol M. (2006). Pro EJB 3: Java Persistence API, Apress, 1590596455, Springer-Verlag New York 233 Spring Street 6th Floor NY 10013

Licari, J. \& Haber, L. (1999). Hybrid Microcircuit Technology Handbook Second Edition, Noyes Publication, 0815514239, 369 Fairview Avenue Westwood New Jersy 07675

Ohring, M. (2002). Materials Science of Thin Films Second Edition, Academic Press, 0125249756, 525 B Street Suite San Diego CA 92101-4495 USA

Whitaker, J. (2005). The Electronics Hand Book Second Edition, CRC Press ***Taylor \& Francis Group, 0849318890, 6000 Broken Sound Parkway NW Boca Raton 